\title{
The Role of Magnetic Reconnection in the Interaction of High-Velocity Clouds and the Galactic Disk
}

\author{
Harald Lesch and Guido T. Birk \\ Institut für Astronomie und Astrophysik, LMU München, Scheinerstr. 1, 81679 \\ München, Germany
}

\begin{abstract}
The boundaries of high-velocity-clouds that hit the Galactic plane are sources of strong $\mathrm{X}$-ray emission indicating gas temperatures of a few million Kelvin. We show that numerous individual magnetic reconnection processes in the interaction region between an HVC and the ionized Reynolds layer may cause the gas heating via dynamical Ohmic dissipation. We present results of numerical plasmaneutral gas fluid simulations performed to study the dynamical reconnection process and to calculate the conversion of magnetic energy into heat.
\end{abstract}

\section{Introduction}

The partially ionized halo plasma is agitated by the activity in the disk (supernova remnants, stellar winds, bubbles and superbubbles, etc.) which results in Galactic fountains (cf. Kahn 1981) formed by hot plasma rising from the Galactic disk. At some height the plasma cools and may partially return to the disk in form of magnetized high-velocity clouds (HVCs) (Kahn 1991). Kerp et al. (1994, 1996), e.g., found clear evidence for the association of HVCs with strong X-ray emission indicating a plasma temperature of a few million degrees. In recent papers we argued that magnetic reconnection can play an important role in the heating of the edges of HVCs. Our analysis was based on the assumption of a fully ionized plasma (Zimmer et al. 1996, 1997). In this contribution we show that the reconnection process also works effectively in a more appropriate weakly ionized regime.

\section{Numerical Model}

We idealize the interaction of an HVC with the halo at a distance of about $1.5 \mathrm{kpc}$ from the disk (cf. Kuntz and Dendy 1996) by considering two magnetized plasmas encountering with a velocity corresponding to the observed HVC-speeds of $100 \mathrm{~km} \mathrm{~s}^{-1}$ (for details see Zimmer et al. 1996). The first stage of the interaction is dominated by compression of the partially ionized plasma and the magnetic field perpendicular to the direction of motion of the cloud. Compression leads to a reversible increase of the plasma pressure 
and temperature in the boundary layer between the cloud and the disk. Due to internal motions inside the layer, the compressed field lines are stretched, twisted, strained and curled by plasma motions. Numerous current sheets will form in the whole interaction volume. The free energy stored in these current filaments can be converted mainly into heat by magnetic reconnection provided that the current densities grow supercritical thereby giving rise to anomalous dissipation (e.g. van Hoven 1985).

We model the impact of a magnetized partially ionized cloud onto the Galactic disk by numerically integrating the continuity, momentum, and energy fluid balance equations (where Ohmic dissipation is included; radiative losses prove to be unimportant) for the plasma and the neutral gas fluid as well as the induction equation (including convective and dissipative terms) that governs the dynamical evolution of the magnetic field (for details of the set of equations and the numerical procedure - an explicit difference scheme based on the Leapfrog algorithm - cf. Birk and Otto 1996). We concentrate on a single current filament to study one elementary reconnection process. The initial magnetic field is chosen as:

$$
\mathbf{B}=B_{0} \tanh \left(z-\frac{z_{\max }+z_{\min }}{2}\right) \mathbf{e}_{y}
$$

A localized resistivity $\eta(j)$ is switched on when the current density, due to the compression caused by the impact, exceeds a critical value.

To model the inflowing plasma of the cloud the magnetic field and the velocities of the ionized $(\mathbf{v})$ and neutral $\left(\mathbf{v}_{\mathbf{n}}\right)$ components at $z=z_{\max }$ are chosen as:

$$
\mathbf{B}=0.9 B_{0} \mathbf{e}_{z} ; \quad \mathbf{v}=\mathbf{v}_{n}=\frac{-v_{0}}{\cosh (y / 10)} \mathbf{e}_{z}
$$

with $v_{0}=0.2$ and $B_{0}=1$ in normalized units. We choose for the plasma particle density, the magnetic field strength and the thickness of the current filament $n_{\text {plasma }}=0.015 \mathrm{~cm}^{-3}, B=17 \mu \mathrm{G}$ and $L=10^{9} \mathrm{~cm}$.

\section{Simulation Results}

Magnetic flux as well as plasma and neutral gas momentum are transported into the $2 \mathrm{D}$ numerical domain with the dimensions $y \in[-15,15]$ and $z \in$ $[0,40]$ in units of $L$. Fig. 1 shows the magnetic field configuration after 60 Alfvénic transit times $\tau_{A}$. Later the initial current sheet becomes supercritical and the resistivity is switched on. The resulting magnetic field configuration after 90 Alfvénic transit times is illustrated in Fig. 2. The reconnection process, easily identified by the occurrence of an X-type configuration located at $y=0$ and $z=32$, works efficiently even in the considered case of a weakly ionized plasma $\left(n_{\text {neutral }} / n_{\text {plasma }}=1000\right)$. During the reconnection process magnetic energy is converted into heat via Ohmic dissipation (cf. Fig. 3). For 
the parameters chosen magnetic flux penetrates too quickly into the interaction region for the reconnection process to dissipate the newly incoming magnetic flux in order to reach a quasistationary state. Consequently, the

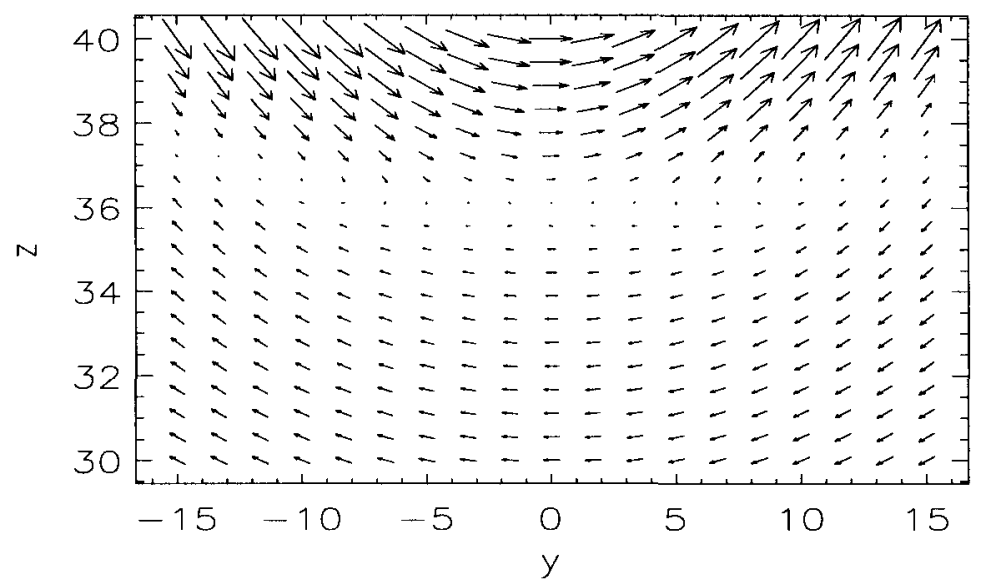

Fig. 1. The magnetic field after $t=60 \tau_{A}$.

overall magnetic energy also grows with time. In the proposed scenario as in the case of a fully ionized plasma regime (Zimmer et al. 1997) plasma should eventually be heated up to some $10^{6} \mathrm{~K}$ during a huge number of individual reconnection processes operating successively at different locations which is in good agreement with observations as in HVC90.5+42.5-130 (Kerp et al. 1994).

Acknowledgements. This work was supported by the DFG through the grant ME 745/18-1.

\section{References}

Birk, G.T., Otto, A. (1996): J. Comp. Phys. 125, 513-525

Kahn, F.D. (ed.) (1981): Dynamics of the Galactic Fountain (Reidel, Dordrecht). Kahn, F. (1991): The Interstellar Disk-Halo Connection in Galaxies, ed. H. Bloemen, IAU Symp. 144, 1-14

Kerp, J., Lesch, H., Mack, K.H. (1994): A\&A 286, L13-16

Kerp, J., Mack, K.H., Egger, R. et al. (1996): A\&A 312, 67-73

Kuntz, K.D., Dendy, L. (1996): ApJ 457, 703-717

van Hoven, G. (1985): Unstable Current Systems and Plasma Instabilities in Astrophysics, eds. M.R. Kundu and G.D. Holman, IAU Symp. 132, 263-271 


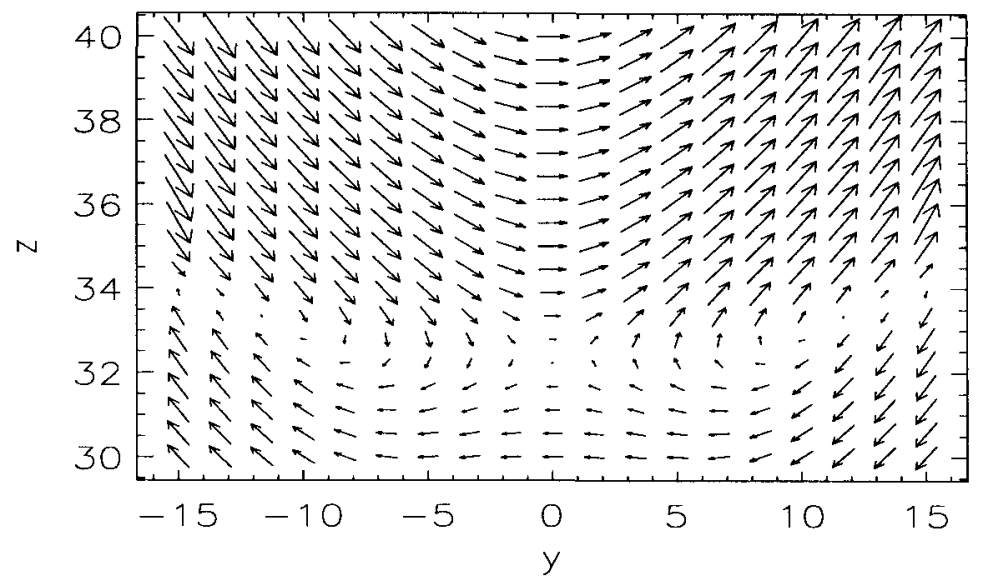

Fig. 2. The magnetic field after $t=90 \tau_{A}$.

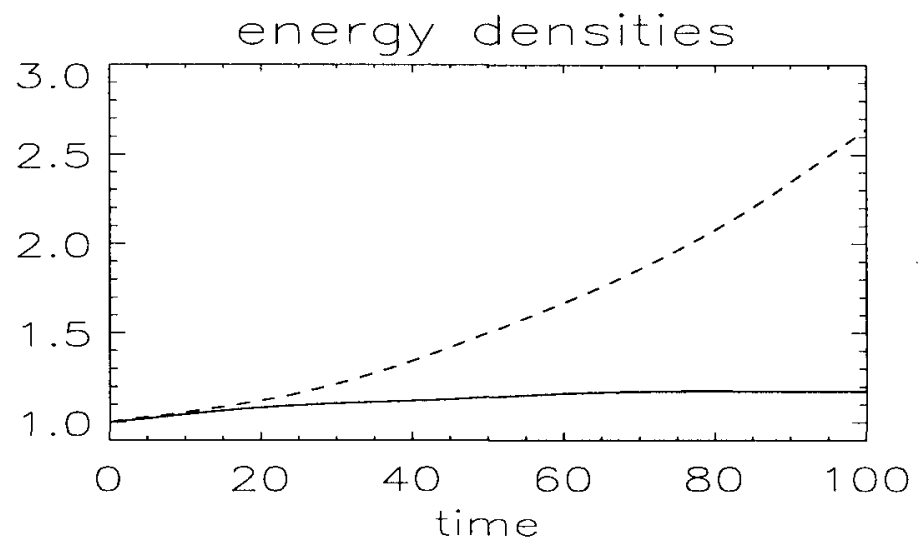

Fig. 3. The temporal evolution (normalized to the respective initial values) of the magnetic field energy density (solid line) and the thermal energy density (dashed line).

Zimmer, F., Birk, G.T., Kerp, J., Lesch, H. (1996): Astrophys. Let. Com. 34, 193-198

Zimmer, F., Lesch, H., Birk, G.T. (1997): A\&A 320, 746-756 